\title{
Correction to: DLX6-AS1/miR-204-5p/OCT1 positive feedback loop promotes tumor progression and epithelial-mesenchymal transition in gastric cancer
}

\author{
Yu Liang ${ }^{1}$ Chun-Dong Zhang ${ }^{1,2,3} \cdot$ Cheng Zhang $^{1} \cdot$ Dong-Qiu Dai ${ }^{1,4}$
}

Published online: 9 November 2021

(c) The International Gastric Cancer Association and The Japanese Gastric Cancer Association 2021

\section{Correction to: Gastric Cancer (2020) 23:212-227 https://doi.org/10.1007/s10120-019-01002-1}

In the original publication of the article, Fig. $2 \mathrm{c}$ and Fig. $4 \mathrm{c}$ were published with errors. The corrected figures are given in this correction.
Fig. 2c. Transwell assays were performed to assess the invasion or migration function of SGC-7901 and AGS cells transfected with si-NC or si-DLX6-AS1
C

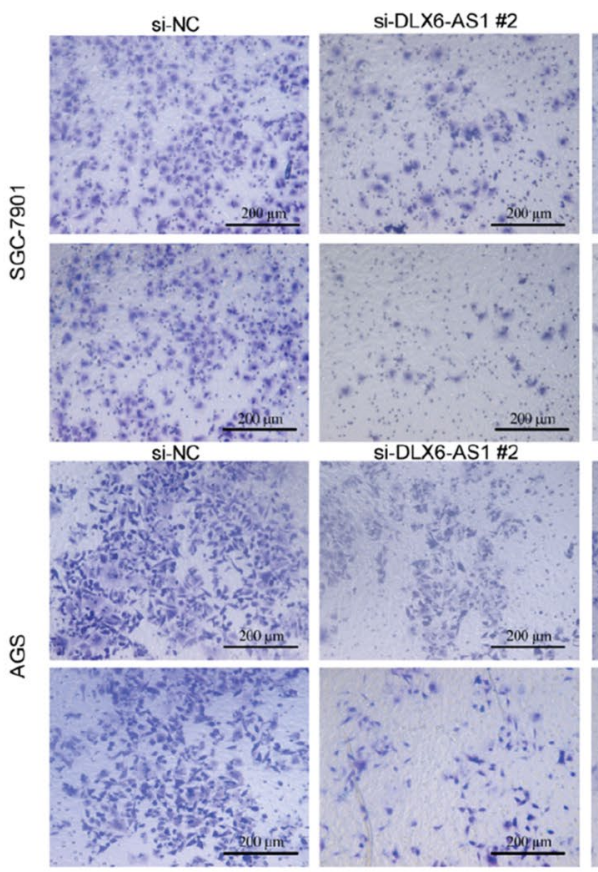

si-DLX6-AS1 \#3
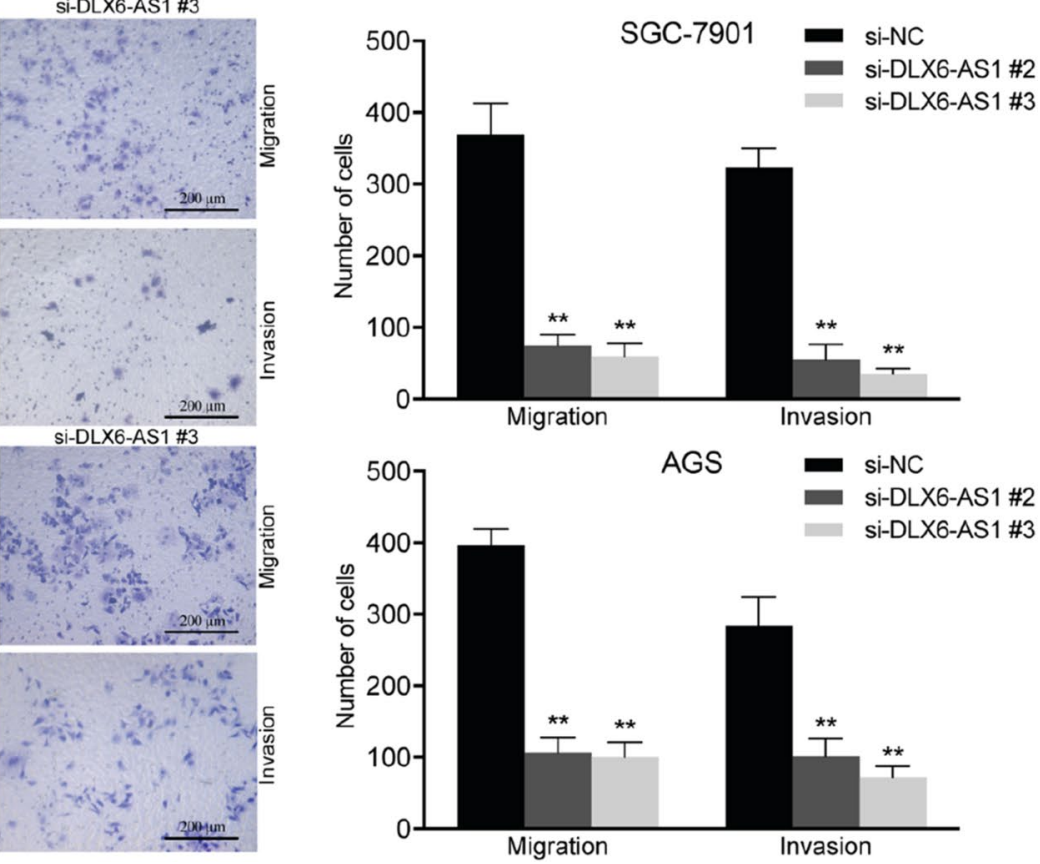

The original article can be found online at https://doi.org/10.1007/ s10120-019-01002-1.

Dong-Qiu Dai

daidq63@163.com

1 Department of Gastrointestinal Surgery, The Fourth Affiliated Hospital of China Medical University, No. 4 Chongshan East Road, Shenyang 110032, China
2 Department of Gastrointestinal Surgery, Graduate School of Medicine, University of Tokyo, 7-3-1, Hongo, Bunkyo-ku, Tokyo 113-0033, Japan

3 Division of Epigenomics, National Cancer Center Research Institute, 5-1-1 Tsukiji, Chuo-ku, Tokyo 104-0045, Japan

4 Cancer Center, The Fourth Affiliated Hospital of China Medical University, No. 4 Chongshan East Road, Shenyang 110032, China 

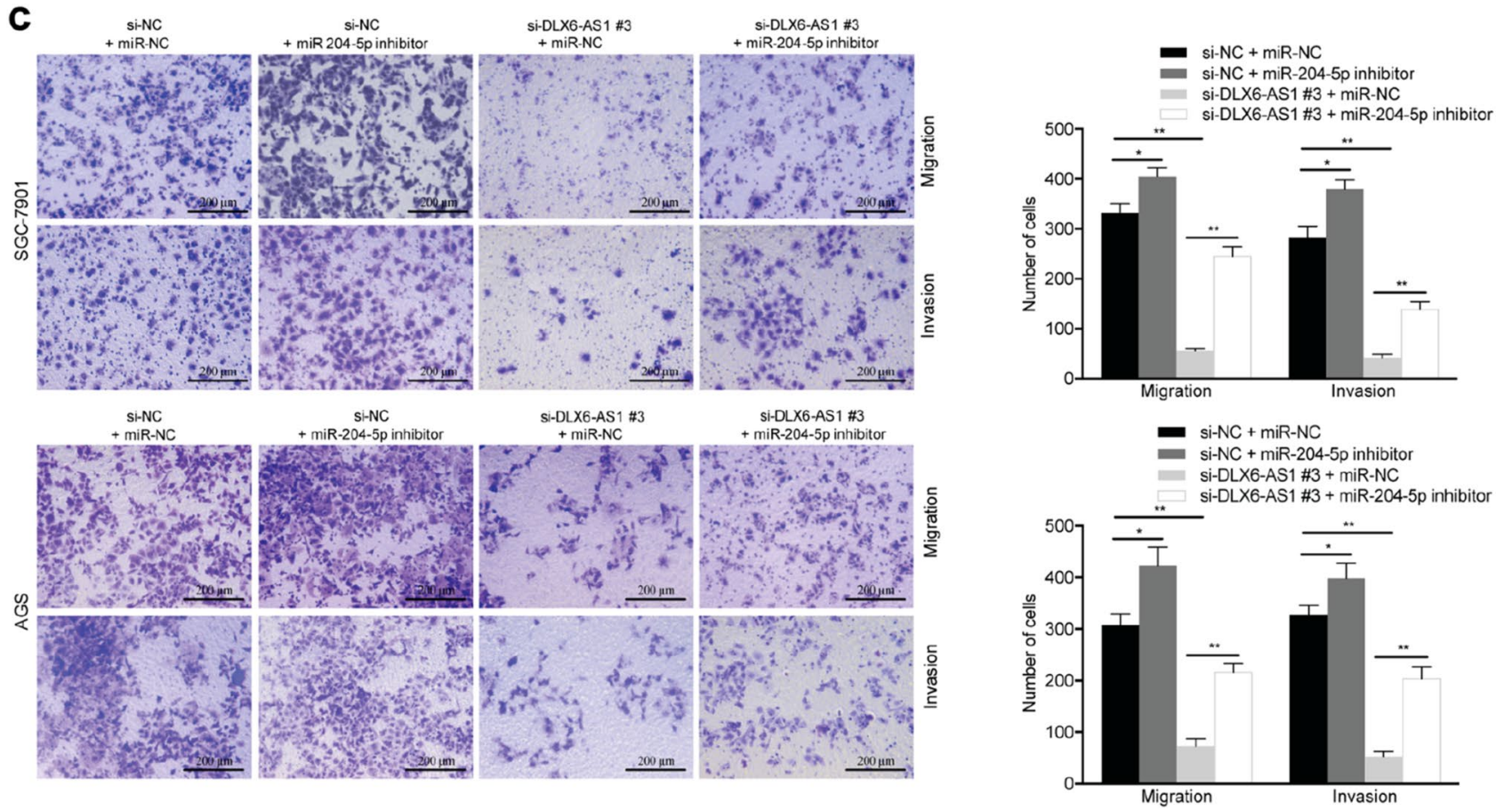

Fig. 4c. Transwell assays were performed to assess the invasion or migration function of SGC-7901 and AGS cells cotransfected with si-NC or si-DLX6-AS1 \#3 and miR-NC or miR-204-5p inhibitor
Publisher's Note Springer Nature remains neutral with regard to jurisdictional claims in published maps and institutional affiliations. 